\title{
Effect of material and structural factors on fracture behaviour of mineralized collagen microfibril using finite element simulation
}

\author{
Abdelwahed Barkaoui \\ SYMME laboratory, Université de Savoie, BP80439, F74944 Annecy-le-Vieux, France \\ Ridha Hambli
}

PRISME laboratory, EA4229, Université d'Orléans, 8 Rue Léonard de Vinci 45072 Orléans,

France

João Manuel R. S. Tavares

Instituto de Engenharia Mecânica e Gestão Industrial, Faculdade de Engenharia, Universidade do Porto, Rua Dr. Roberto Frias, 4200-465 Porto, Portugal

\section{Corresponding author:}

Professor João Manuel R. S. Tavares

Departamento de Engenharia Mecânica

Faculdade de Engenharia da Universidade do Porto

Rua Dr. Roberto Frias

4200-465 Porto

Portugal

Phone: +351 225081 487, Fax: +351 225081445

Email: tavares@fe.up.pt, url: www.fe.up.pt/ tavares 


\title{
Effect of material and structural factors on fracture behaviour of mineralized collagen microfibril using finite element simulation
}

\begin{abstract}
Bone is a multiscale heterogeneous material and its principal function is to support the body structure and to resist mechanical loads without fracturing. Numerical modelling of biocomposites at different length scales provides an improved understanding of the mechanical behaviour of structures such as bone, and also guides the development of multiscale mechanical models. Here, a three-dimensional nano-scale model of mineralized collagen microfibril based on the finite element method was employed to investigate the effect of material and structural factors on the mechanical equivalent of fracture properties. Fracture stress and damping capacity as functions of the number of cross links were obtained under tensile loading conditions for different densities and Young's modulus of the mineral phase. The results show that the number of cross-links and the density of mineral as well as Young's modulus of mineral have an important influence on the strength of mineralized collagen microfibrils which in turn clarify the bone fracture on a macro-scale.
\end{abstract}

Keywords: nano-scale model; cross-links; finite element method; fracture mechanical properties 


\section{INTRODUCTION}

Bone is a multiscale material with a very complicated hierarchical structure. This hierarchical structure has different scales or levels, specific interactions between these levels and a highly complex architecture in order to fulfil bone biological and mechanical functions (Barkaoui and Hambli, 2011; Sergey, 2010). Katz et al. and Feng et al. (Katz et al.,1987; Feng et al., 2010) divide the hierarchical structure into five levels that have been widely accepted in the scientific community: (i) a Nano structural level (ranging from a few nanometres to several hundred nanometres) - bone at this level can be considered as a multi-phase nano-composite material consisting of an organic phase (32 - 44\% of bone volume), an inorganic phase (33 $43 \%$ of bone volume), and water (15 - 25\% of bone volume); (ii) a Sub-micro-structural level, also called a single lamella level (spanning from one to a few microns); (iii) a Microstructural level (from tens to hundreds of microns), or a single osteon and an interstitial lamella level; (iv) a Meso-structural level (from several hundred microns to several millimetres), or the cortical bone level; and finally, (v) a Macro-structural level, or whole bone level (several millimetres to several centimetres, depending on the species).

Bone strength is governed by the characteristics of collagen, including the collagen crosslinks that have an important role in the reinforcement of bone strength. The biomechanical effects of collagen depend largely on the cross-linking (Knott and Bailey, 1998; Viguet-Carrin, 2006). The strength and stability during maturation of the microfibrils are attained by the development of intermolecular cross-links (Stančíková et al., 1999). Experimental evidence (Allen et al., 2008; Wu et al., 2003; Boxberger and Vashishth, 2004) has shown that collagen cross-linking in bone tissue significantly influences its deformation and failure behaviour. Additionally, experiments in vitro (Wu et al., 2003; Boxberger and Vashishth, 2004) and in vivo (Viguet-Carrin, 2006a; Allen et al., 2008; Wu et al., 2003) revealed that an increases in the number of cross-links is associated with the enhancement of certain mechanical properties (strength and stiffness) and a reduction in others (energy absorption). There are two types of cross-links: enzymatically and non-enzymatically (Seigmund et al., 2008). Considering the macroscopic response of bone, enzymatic cross-linking has been related to improving mechanical properties (Banse et al., 2002) whereas non-enzymatic cross-linking prevents energy absorption by micro damaged formations and may accelerate brittle fracturing (Vashishth et al., 2004; Tang et al., 2007; Nyman et al., 2007; Vashishth, 2007). Natural cross-linking gives collagen a high tensile strength and proteolytic resistance (Friess, 1998). 
Nano-scale failure properties of the mineralized collagen microfibril were studied here and the mechanical and structural parameters of all phases analysed. The cross-linking is one of the most important structural elements of type I collagen in mineralized tissues that provides the fibrillar structure and contributes to various mechanical properties, such as tensile strength, fracture toughness and viscoelasticity (Elham and Iwona, 2012). Fritsch et al., (Fritsch et al., 2009) used the multiscale micromechanics elastic theory in an elasto-plastic analysis to predict the strength of cortical bone. These authors found that the failure of bone material initiates at the nano-scale by a ductile sliding of hydroxyapatite (HA) crystals along layered water films, followed by a rupture of the collagen cross-links. Siegmund et al., (Seigmund et al., 2008) proposed a model that addresses the effect of collagen cross-linking on the mechanical behaviour of a mineralized collagen fibril. Buehler (Buehler, 2008) extended a Molecular Dynamics Model of a single collagen molecule to an individual collagen fibril to obtain its mechanical response under uniaxial tension. The results showed that the deformation and failure mechanisms of a collagen fibril are strongly influenced by the crosslinking density. Barkaoui and Hambli (Barkaoui and Hambli, 2011) proposed a threedimensional (3D) finite element method (FEM) model to represent the structure of the mineralized collagen microfibril with three constituents: mineral, tropocollagen (TC) molecules and cross-links. This model was used to study the elastic and mechanical failure behaviour of mineralized collagen microfibril under a varying number of cross-links, based on an array of five collagen molecules that are cross-linked together by spring elements and embedded in a mineral matrix.

In this study, the 3B FEM model proposed by Barkaoui and Hambli $(2011,2012)$ was enhanced to allow dapping and fracture stress calculations, which were used to study the failure of mineralized collagen microfibril as well as the biomechanical effect of the constituent properties on the fracture behaviour. The enhanced 3D FEM model of mineralized collagen microfibril proved to be a good solution for bottom-up investigations on structureproperty relationships in human bone.

\section{METHODS AND TOOLS}

\subsection{Mineralized collagen microfibril}

The existence of sub-structures in collagen fibrils has been a topic of extensive debate. Recent studies have suggested the presence of microfibrils in fibrils (Yang et al., 2012). Experimental 
work conducted by Orgel, Fratzl and others have proved that all collagen-based tissues are organized into hierarchical structures, where the lowest hierarchical level consists of triple helical collagen molecules (Fratzl, 2008; Orgel et al., 2006; Orgel et al., 2001) and the multiscale structure is defined as TC/fibrils/fibres. Also, other authors have observed a longitudinal microfibrillar structure with a width of 4 - $8 \mathrm{~nm}$ (Habelitz et al., 2002; Baselt et al., 1993). Three-dimensional image reconstructions of $36 \mathrm{~nm}$-diameter corneal collagen fibrils also showed a $4 \mathrm{~nm}$ repeat in a transverse section that was related to the microfibrillar structure (Holmes et al., 2001). Using X-ray diffraction culminating in an electron density map, Orgel et al. (Orgel et al., 2001) suggested the presence of right-handed super-twisted microfibrillar structures in collagen fibrils. The microfibril is a helical assembly of five TC molecules that offset one another with an apparent periodicity of $67 \mathrm{~nm}$. These five molecules create a cylindrical formation with a diameter of 3.5-4 nm however its length is unknown. Smith (Smith, 1968) suggested that each microfibril consists of exactly five molecules in a generic circular cross section (Figure 1). Lee et al., (Lee et al., 1996) suggested that collagen microfibrils have a quasi-hexagonal structure. Barkaoui and Hambli (Barkaoui and Hambli, 2011) also used the cylindrical representation of Smith's microfibril model (Figure 1) to develop their 3D finite element model of microfibrils. This choice was based on the fact that the mechanical behaviour of microfibrils under tensile load depends mainly on the area of the cross section, while the shape has no influence under tensile load functions.

[Insert Figure 1 about here]

As aforementioned, bone is a complex composite material consisting mainly of collagen (TC molecules) and mineral (HA crystals). Figure 2 illustrates that collagen is a very resistant material that give bone its toughness. The Figure also depicts that the mineral HA is very rigid, which gives bone its rigidity and resistance to fracture. As such, the mechanical properties of bone depend on the characteristics of its basic components and the interaction between them (Li et al., 2003; Walsh and Guzelsu, 1994).

[Insert Figure 2 about here]

The fundamental components of the microfibril can be distinguished as follows: 
(a) Collagen: Long cylindrically shaped TC molecules establishing a continuum in which mineral crystals are embedded. Lees and Fratzl et al. (Lees, 1981; Fratzl et al., 1993) reported that the effective molecular diameter of dry TC molecules is $1.09 \mathrm{~nm}$ and that of wet TC molecules is about 1.42-1.5 nm. Hydration affects the packing, as molecules are closer, and the sliding between molecules becomes more difficult due to increased adhesion. The length of TC molecules is about $300 \mathrm{~nm}$ (Buehler, 2008; Gautieri et al., 2011), and they are selfassembled in the form of microfibrils.

(b) Mineral: Plate or needle-shaped mineral crystals consisting of impure $\mathrm{HA}\left(\mathrm{Ca}_{10}\left[\mathrm{PO}_{4}\right]_{6}\right.$ $[\mathrm{OH}]_{2}$ ) with a typical thickness of 1-5 nm, and length of 25-50 nm (Weiner and Wagner, 1998). This lengthiest dimension is typically found to be oriented parallel to the collagen molecules. As reported by some authors, HA mineral is stiff and extremely fragile, exhibiting elastic isotropic behaviour (Seigmund et al., 2008; Pidaparti et al, 1996).

The mechanical elastic properties of the TC molecules and the mineral phase used in this study were taken from the literature. It should be noted that a wide range of values has been proposed in different studies: with the TC molecule values ranging from 0.35 to $12 \mathrm{GPa}$ (Gautieri et al., 2011; Sun et al., 2002) and from 62.75 to $170 \mathrm{GPa}$ for HA mineral (Weiner and Wagner, 1998; Cowin, 1989; Currey, 1969). The failure properties of TC molecules and mineral reported in literature (Akao et al., 1981; Shareef et al., 1993) are shown in Table 1.

[Insert Table 1 about here]

Researchers have also used different values of volume fraction for the mineral crystals, varying from 32 to 52\% (Nikolov and Raabe, 2008). Some values of the mineral volume fraction reported in the literature are listed in Table 2.

[Insert Table 2 about here]

(c) Different non-collagenous organic molecules: These are predominantly lipids and proteins that regulate HA mineralization, probably by proteins supporting or inhibiting mineralization, and possibly also by lipids (Urist et al., 1983).

(d) Water: Provides the liquid environment for the biochemical activity of the noncollagenous organic matter. Water at ultrastructure level of bone exists in the form of bound 
water in the collagen network, including the collagen-mineral interface, and tightly bound water in the mineral phase (Wang and Puram, 2004).

(e) Cross-links: Cross-linking of TC molecules (Figure 2) plays a critical role in bone microfibrils, fibrils and fiber connectivity (Fratzl and Weinkamer, 2007; Gelse et al., 2003; Gupta et al., 2003; Wang et al., 2002). The formation of these cross-links is mediated by the enzyme lysine oxidase (Eyre et al., 2008). The formation of intermolecular covalent crosslinks has significant effects on the material properties, mainly on the strength and brittleness (Buehler, 2008), and on the mechanical behaviour (Bailey, 2001; Saito and Marumo, 2009). To study the mechanical behaviour of mineralized collagen microfibril, Barkaoui and Hambli (Barkaoui and Hambli, 2011) used the rheological model (Figure 3(c)) proposed by Uzel and Buehler (Uzel and Buehler, 2011) to model the cross-links by the FEM.

As the present work focused on the elastic regime of mineralized collagen microfibril and initiation of fracture, only the elastic regime (Figure 3 (c)) was taken into consideration. Additionally, linear spring elements with stiffness $k_{c r}=1181.143 \mathrm{e}-11 \mathrm{~N} / \mathrm{nm}$ (Barkaoui and Hambli, 2013) were integrated into the model in order to attain the FEM behaviour of a crosslink driven by a constitutive model.

[Insert Figure 3 about here]

\subsection{D FEM model}

Barkaoui and Hambli (Barkaoui and Hambli, 2013; Hambli and Barkaoui, 2012) used a 3D model with a cylindrical form of the mineralized collagen microfibril to perform a FEM analysis in ABAQUS (Dassault Systèmes, USA) software. The 3D geometry model was meshed using tetrahedral elements and solved via the ABAQUS standard scheme (Barkaoui and Hambli, 2013). For the boundary conditions, it was assumed that the mineralized collagen microfibril retains its cylindrical form during elastic loading. The left surface of the microfibril was encastred and a uniaxial force $(F)$ along the axis of the collagen molecules was applied to the right surface of the microfibril. Deformation and elongation $\Delta l$ were computed.

The original Barkaoui and Hambli's modelling of mineralized collagen microfibril was improved here in order to calculate the damping capacity and fracture stress. 


\subsection{Microfibril fracture evaluation}

The current study focuses on the elastic and fracture behaviour of the mineralized collagen microfibril.

An approach to calculate the apparent tensile stress $\sigma_{a p p}$ and the corresponding strain $\varepsilon_{a p p}$ applied to the microfibril is to consider:

$$
\sigma_{\text {app }}=\frac{F}{A}
$$

and

$$
\varepsilon_{\text {app }}=\frac{\Delta l}{l_{0}}
$$

where $A, \Delta l$ and $l_{0}$ are the apparent area of the microfibril, the total microfibril elongation computed by the FEM analysis and the initial length of the microfibril, respectively.

The HA crystals which gives the bone its stiffness and resistance are much stronger than the TC molecules. Thus, the fracture of mineral is directly associated to the rupture of the mineralized collagen microfibril. As the tensile strength of HA crystals is $\sigma_{\mathrm{HA}}^{u l t, t}=52.2 \mathrm{MPa}$, we considered that the mineralized collagen microfibril is broken when $\sigma_{\text {app }}$ reaches this value. As such, the stress fracture was calculated as:

$$
\sigma_{R}(\text { structure })=\frac{F_{R}}{A},
$$

where force $F_{R}$ corresponds to the force that leads to a von Mises stress in HA mineral higher than or equal to the tensile strength of HA mineral, and $A$ is the microfibril section.

\subsection{Mineral-collagen interface}

Previous numerical studies using mineral-collagen interface modelling have indicated that the interfacial de-bonding of the mineral and collagen phases may have a significant influence on the pattern of micro damage progression, i.e., linear microcracks or diffuse damage, in bone 
under tensile deformation but not on fracture initiation (Dong et al., 2009). Several authors have developed FEM models to investigate the influence of the mineral-collagen interface on a fracture of mineralized collagen fibril (Qing et al., 2011; Dong et al., 2010). The simulations conducted were generally based on cohesive elements to model the de-bonding and sliding behaviour observed for the initiation of the fracture at the interface. In the current investigation, only the first stage of the fracture was considered; that is, the fracture initiation. When the mineral reaches its critical value of fracture, fracturing of the microfibril takes place. Sliding at the mineral and collagen interface may occur prior and/or after the mineral cracking, which can affect the softening of the mineralized collagen fibril (Siegmund et al., 2008; Buehler, 2008). Here, we focused on the role of cross-links in the fracture properties of mineralized collagen microfibril, and therefore, the effects of the mineral-collagen interface could be neglected.

\subsection{Damping capacity}

The damping capacity expresses the facility that a material can dissipate vibrational energy (Figure 4). We applied this principle to study the effect of cross-links on the damping capacity of the bone on the nano-scale.

[Insert Figure 4 about here]

An elastically loaded material with a constraint $\sigma$ stores energy per volume unit given as:

$\int_{0}^{\varepsilon} \sigma d \varepsilon=\frac{1}{2} E \varepsilon^{2}$

and in a cycle of loading and unloading, it dissipates energy formulated as:

$$
\Delta \mathrm{U}=\oint \sigma \mathrm{d} \varepsilon
$$

Therefore, the damping capacity was calculated here as:

$$
\eta=\frac{\Delta U}{2 \pi U}
$$


which was calculated using the analytic expression:

$\eta=\frac{\sigma_{\max }(N)-\sigma_{\text {max }}(N=0)}{2 \pi \sigma_{\max }(N=0)}$,

where $N$ is the number of cross-links, $\sigma_{\max }(N)$ the maximum stress given by the FEM simulation for the different number of cross-links, and $\sigma_{\max }(N=0)$ is the maximum stress of the microfibril without cross-links.

\section{RESULTS AND DISCUSSION}

This study aimed to investigate the influence of the number of cross-links, Young's modulus of HA mineral $\left(E_{m}\right)$ and HA mineral volume fraction $\left(V_{m}\right)$ on the failure properties of mineralized collagen microfibril. The improved FEM model was used under small strain ( $\varepsilon<5 \%$ ) with a well-defined configuration in order to validate the results and data available in terms of characterization of the ultrastructure of bone under tension load.

Two cases were addressed: wet collagen and dry collagen. Figure 5 illustrates the stress-strain curves obtained for the FEM model configuration corresponding to $E_{m}=114 \mathrm{GPa}$ and a constant Young's modulus of the collagen phase $E_{c}=2.7 \mathrm{GPa}$.

[Insert Figure 5 about here]

A very close agreement was found for hydrated collagen microfibril in the small strain regime between the FEM based results and experimental ones obtained by X-ray diffraction (Sasaki and Odajima, 1996) and also by atomic force microscopy (AFM) (Van der Rijt et al. 2006; Aladin et al., 2010).

To study the influence of Young's modulus of HA mineral on the fracture behaviour of the microfibril, we chose a constant Young's modulus of the collagen phase $E_{c}=2.7 \mathrm{GPa}$ and constant mineral volume fraction of $V_{m}=48 \%$. 
Figure 6 depicts that the equivalent fracture stress of the microfibril depends on Young's modulus of the mineral phase and the number of cross-links. It should be noted that, as we are dealing with a complex and living material, the values found are approximate and depend on several factors, such as age, phase properties, number of cross-links, geometry, bone mineral density BMD, type of collagen and disease.

Figure 7 clarifies that the damping capacity of the microfibril depends on the number of crosslinks. On the contrary, the effect of Young's modulus of HA mineral revealed to be insignificant.

[Insert Figures 6 and 7 about here]

Figure 8 illustrates that Von Mises stress increases when the number of cross-links increases, which means that the microfibril becomes more rigid and resistant.

[Insert Figure 8 about here]

In the second investigation, the improved FEM model was used to study the influence of the mineral volume fraction of phase and of the number of cross-links on the failure behaviour of the microfibril. Three FEM models with different distribution phases were built. The idea was to vary the TC molecule diameter from 1.2 to $1.5 \mathrm{~nm}$, with Young's modulus of the two constant phases $\left(E_{m}=114 \mathrm{GPa}\right.$ and $\left.E_{c}=2.7 \mathrm{GPa}\right)$.

Figures 9 and 10 demonstrate that the equivalent fracture stress and damping capacity depend largely on both mineral volume fraction and the number of cross-links.

[Insert Figures 9 and 10 about here]

Figure 11 depicts the initiation of the microfibril cracking with the variation of the number of cross-links. This Figure suggests that the number of cross-links affects the acceleration of cracking, which can be explained by the fact that as the number of cross-links increases, the microfibril rigidity increases.

[Insert Figure 11 about here] 
The graphs in Figures 6, 7, 9 and 10, show that the number of cross-links has the highest influence on the increase of the fracture stress. Increasing the number of cross-links leads to a significant non-linear increase in the microfibril stiffness. However, when $N>20$, the fracture stress of microfibril and the damping capacity do not depend on the number of crosslinks, and they remain at a constant value or "plateau value". The same phenomenon was also observed by Buehler (Buehler, 2008) using Molecular Dynamic (MD) computation. Buehler found that the yield and the fracture stress depend on the cross-link density $\beta$. The plateau value that was obtained by our findings is explained by a change in the molecular deformation mechanism from predominantly shear (when $\beta<25$ ) to molecular fracture (when $\beta>25$ ).This plateau value can also be explained by the fact that whenever the number of cross links increases, their stiffness increases up to a threshold value at which the behaviour of all collagen cross-links becomes insensitive to this number of cross links to any further increase. In the macroscopic response of bone, cross-links have been coupled to improve mechanical properties (Banse et al., 2002) and prevent energy absorption by micro-damage formation and may accelerate brittle fracture (Tang et al., 2007; Nyman et al., 2007). The same results were found here for the nano-scale collagen microfibril.

Studies in vitro (Viguet-Carrin et al., 2006; Wu et al., 2003) and in vivo (Allen et al., 2008) have reported that increases in the cross-linking numbers are associated with enhancement of some mechanical properties and reductions of others. These experimental data are limited in their ability to define individual biomechanical effects of altered cross-linking numbers (Buehler, 2008). The study of the effect of cross-links conducted here is more precise and realistic because the used FEM model success to combines the collagen, mineral and crosslinks. Moreover, the improved model is able to varying the mechanical and geometry properties of each phase and to foresee their influence on the corresponding equivalent properties of the collagen microfibril.

This study also shows that collagen cross-linking in bone tissue significantly affects the bone mechanical properties. For example, an increase in the amount of cross-linking is not only associated to an improvement in strength and stiffness, but also to a reduction of energy absorption and ductility. The microfibril structure is stabilized through intermolecular crosslinks joining two TC molecules. Mutations in collagen structure and changes in collagen cross-linking pattern have been observed in some bone diseases such as osteoporosis and 
osteogenesis (Elham and Iwona, 2012). The challenge is to define which parameters to use to capture the actual nature of cross-linking of collagen molecules in healthy or diseased bone.

\section{CONCLUSION}

Here, the nano-mechanical properties at microfibril failure were investigated using 3D FEM simulation. Regarding the macroscopic scale, consistent and similar results to the ones found in the literature were achieved. The findings here demonstrate the important role of crosslinks for good mechanical properties and the prevention of energy absorption, which increases the damping capacity. This work also gives an improved understanding of bone on a nanoscale and a closer look at the upper level scale which is the collagen fibril.

With the 3D improved FEM model we aimed to model the microfibril realistically, but this model is still limited owing to the specific arrangement of collagen molecules, the orientation of mineral crystals, the complex distribution of each phase and in the manner of modelling the two types of cross-links. Besides, the model does not take into account the mineral-collagen interface, which is also an issue to study in the near future.

\section{ACKNOWLEDGEMENTS}

This work was supported by the French National Research Agency (ANR) through the TecSan program (Project MoDos, nºAN-09-TECS-018).

\section{REFERENCES}

Akao, M., Aoki, H., Kato, K..1981. Mechanical properties of sintered hydroxyapatite for prosthetic applications. Journal of Materials Science 16(3):809-812.

Aladin, D. M., Cheung, K. M., Ngan, A. H., Chan, D., Leung, V. Y., Lim, C. T., Luk, K. D., Lu, W. W. 2010.Nanostructure of collagen fibrils in human nucleus pulposus and its correlation with macroscale tissue mechanics., J. Orthop. Res 28(4):497-502.

Allen, M.R., Gineyts, E., Leeming, D.J., Burr, D.B., Delmas, P.D. 2008. Bisphosphonates alter trabecular bone collagen cross-linking and isomerization in beagle dog vertebra. Osteoporosis International, Osteoporos Int.19(3):329-337.

Andreas, F., Luc, D.,Christian, H., Sanahuja, J. 2009. Mechanical behaviour of hydroxyapatite biomaterials, an experimentally validated micromechanical model for elasticity and strength, journal of biomedical Materials research Part A 88A(1):149161. 
Bailey, A.J., Paul, R.G., Knott, L. 1998. Mechanisms of maturation and ageing of collagen. Mech. Age. Dev106(1-2):1-56.

Bailey, AJ. 2001. Molecular mechanisms of ageing in connective tissues. Mechanisms of Ageing and Development 122(7):735-755.

Banse, X., Sims, T.J., Bailey, A.J. 2002. Mechanical properties of adult vertebral cancellous bone: correlation with collagen intermolecular cross-links. Journal of Bone and Mineral Research 17(9):1621-1628.

Barkaoui, A. and Hambli, R. 2013.Nanomechanical properties of mineralised collagen microfibrils based on finite elements method: biomechanical role of cross-links, Computer Methods in Biomechanics and Biomedical Engineering,DOI:10.1080/10255842.2012.758255.

Barkaoui, A., Hambli, R. 2011. Finite element 3D modeling of Mechanical Behaviour of Mineralized collagen Microfibril, J ApplBiomaterBiomech 9(3): 207-213.

Baselt, D.R., Revel, J.P., Baldschwieler, J.D. 1993. Subfibrillar structure of type I collagen observed by atomic force microscopy. Biophys. J. 65(6):2644-2655.

Boxberger, J., Vashishth, D. 2004. Non enzymatic glycation affects bone fracture by modifying creep and inelastic properties of collagen. Transactions of the Orthopaedic Research Society 29:0491.

Buehler, M.J. 2008.Nanomechanics of collagen fibrils under varying cross-link densities: atomistic and continuum studies. J. Mech.Behaviour Biomed.Mater. 1(1):59-67.

Cowin, S.C.1989. Bone Mechanics. CRC Press, Boca Raton, 255-255.

Currey, J. D. 1969.The relationship between the stiffness and the mineral content of bone. J. Biomech. 2(4):477-480.

Dong,X.N., Teja, G., Harry, R.M., Xiao, D.W. 2009. Probabilistic failure analysis of bone using a finite element model of mineral-collagen composites, Journal of Biomechanics 42(3):202-209

Dong, X.N., Luo, Q., Sparkman, D., Millwater, H., Wang, X. 2010.Random field assessment of nanoscopic in homogeneity of bone. Bone 47:1380-1384.

Elham,H., Iwona, J. 2012. Elastic modeling of bone at nanostructural level. Materials Science and Engineering R 73: 27-49.

Eyre, D.R., Weis, M.A., Wu, J.J. 2008. Advances in collagen crosslink analysis. Methods 45 (1):65-74.

Feng, L. 2010. Multi-scale characterization of swine femoral cortical bone and long bone defect repair by regeneration. Ph.D. dissertation, University of Illinois at UrbanaChampaign.

Fratzl, P., Weinkamer, R. 2007.Nature’s hierarchical materials. Progress in Materials Science 52:1263-1334.

Fratzl, P. 2008. Collagen: Structure and Mechanics. Springer (New York).

Fratzl, P., Fratzl-Zelman, N., Klaushofer, K.1993. Collagen packing and miner- alization. An X-Ray scattering investigation of Turkey leg tendon.Biophys. J. 64:260-266.

Friess, W. 1998. Collagen - biomaterial for drugdelivery. Eur J Pharm Biopharm 45:113-136.

Gautieri, A., Vesentini, S.,Redaelli, A., Buehler,M.J. 2011.Hierarchical structure and nanomechanics of collagen microfibrils from the atomistic scale up, Nano Letters 9, 11(2):757-66.

Gelse, K., Poschl, E., Aigner, T. 2003.Collagens-structure, function, and biosynthesis. Advanced Drug Delivery Reviews 55(12):1531-1546.

Gupta,H.S., Seto, J., Krauss, S., Boesecke, P., Screen H.R.C. 2010. In situ multi-level analysis of viscoelastic deformation mechanisms in tendon collagen. Journal of Structural Biology 169(2):183-191. 
Habelitz, S., Balooch, M., Marshall, S.J., Balooch, G., Marshall, G.W. 2002.In situ force microscopy of partially demineralized human dentin collagen fibrils. J. Struct. Biol 138:227-236.

Hambli, R., Barkaoui, A. 2012.Physically based 3D finite element model of a single mineralized collagen microfibril, Journal of Theoretical Biology 301:28-41.

Holmes, D.F., Gilpin, C.J., Baldock, C., Ziese, U., Koster, A.J., Kadler, K.E.2001. Corneal collagen fibril structure in three dimensions: structural insights into fibril assembly, mechanical properties, and tissue organization. Proc. Natl. Acad. Sci. USA 98:73077312.

Jager, I., Fratzl, P. 2000. Mineralized collagen fibrils: a mechanical model with a staggered arrangement of mineral particles. Biophys. J. 79:1737-1746.

Ji, B., Gao, H. 2006. Elastic properties of nanocomposite structure of bone. Compos. Sci. Technol. 66:1209-1215.

Katz, J.L., Meunier, A. 1987.The elastic anisotropy of bone. J. Biomech. 20, 1063-1070.

Knott, L., Bailey, A.J.1998. Collagen cross-links in mineralizing tissues: a review of their chemistry, function, and clinical relevance. Bone 22:181-187.

Kotha, S.P., Guzelsu, N. 2000. The effects of interphase and bonding on the elastic modulus of bone: changes with age-related osteoporosis. Med. Eng. Phys. 22.

Yang. L., van der Werf. K.O., DijkstraP.J., J. Feijen, M.L. 2012. Bennink, Micromechanical analysis of native and cross-linked collagen type I fibrils supports the existence of microfibrils; Journal of the mechanical behavior of biomedical materials.6:148-158.

Lee, J., Scheraga, H.A., Rackovsky, S. 1996. Computational study of packing a collagen-like molecule: quasi-hexagonal vs "Smith" collagen microfibril model. Biopolymers 40(6):595-607.

Lees, S. 1981. A mixed packing model for bone collagen. Calcif. Tissue Int 33(6):591-602.

Lees, S. 1987. Considerations regarding the structure of the mammalian miner- alized osteoid from viewpoint of the generalized packing model. Connect. Tissue Res 16:281-303.

Li, X., Agrawal M., Wang X. 2003.Age dependence of in situ thermostability of collagen inhuman bone”.Calcif.Tissue.Int 72:513-518.

Nikolov, S., Raabe, D. 2008. Hierarchical modeling of the elastic properties of bone at submicron scales: the role of extra fibrillar mineralization. Biophys. J. 94:4220-4232.

Nyman, J.S., Roy, A., Tyler, J.H., Acuna, R.L., Gayle, H.J., Wang, X. 2007.Age-related factors affecting the post yield energy dissipation of human cortical bone. Journal of Orthopaedic Research 25: 646-655.

Orgel, J.P.R.O., Miller. A., T.C. Irving, R.F. Fischetti, A.P. Hammersley, and T.J. Wess; 2001. TheIn Situ Supramolecular Structure of Type I Collagen. Structure 9:1061-1069.

Orgel, J.P.R.O., T.C. Irving, A. Miller, and T.J. Wess. 2006. Microfibrillar structure of type I collagen in situ. P. Natl. Acad. Sci. USA, 103(24):9001-9005.

Pidaparti, R.M., Chandran, A., Takano, Y., Turner, C.H. 1996. Bone mineral lies mainly outside collagen fibrils: predictions of a composite model for osteonal bone. J. Biomech 29:909-916.

Luo, Q., Nakade, R., Dong, X., Rong, Q., Wang, X. 2011. Effect of mineral-collagen interfacial behavior on the microdamage progression in bone using a probabilistic cohesive finite element model, Journal of the mechanical behavior of biomedical materials 4:943-952.

Rho,J.Y., Kuhn-Spearing, L., Zioupos, P. 1998. Mechanical properties and the hierarchical structure of bone. Med.Eng.Phys 20:92-102.

Saito, M., Marumo, K. 2009. Collagen cross-links as a determinant of bone quality: a possible explanation for bone fragility in aging, osteoporosis, and diabetes mellitus. Osteoporosis International 21(2):195-214. 
Sasaki, N., Odajima, S. 1996.Stress-strain curve and Young's modulus of a collagen molecule as determined by the X-ray diffraction technique. J. Biomech 29:655-658.

Sasaki, N., Ikawa, T., Fukuda, A. 1991.Orientation of mineral in bovine bone and the anisotropic mechanical properties of plexiform bone. J. Biomech. 24:57-61.

Dorozhkin,S.V. 2010.Nanosized and nanocrystalline calcium orthophosphates, ActaBiomaterialia 6:715-734.

Shareef, M.Y., Messer, P.F., van Noort, R. 1993. Fabrication, characterization and fracture study of a machinable hydroxyapatite ceramic. Biomaterials 14(1):69-75.

Siegmund, T., Allen, M.R., Burr, D.B. 2008. Failure of mineralized collagen fibrils: modeling the role of collagen cross-linking. Journal of Biomechanics 41:1427-1435.

Smith, J.W. 1968. Molecular pattern in native collagen. Nature 219:157-158.

Stančíková, M., Stančík, R., Gubzová, Z., Rovenský, J. 1999.Collagen in the Treatment of Rheumatic Diseases - Oral Tolerance, Bratislavskélisty.

Sun, Y., Luo, Z., Fertala, A., An K.2002.Direct quantification of the flexibility of type Icollagen monomer, Biochemical and Biophysical Res. Com., 295(2):382-386.

Tang, S., Zeenath, U., Vashishth, D. 2007.Effects of non-enzymatic glycation on cancellous bone fragility. Bone 40:1144-1151.

Urist, M.R., DeLange, R.J., Finerman, G.A.M.1983.Bone cell differentiation and growth factors. Science 220:680-686.

Uzel,SGM., Buehler, M.J. 2011. Molecular structure, mechanical behaviour and failure mechanism of the C-terminal cross-link domain in type I collagen. Journal of the Mechanical Behaviour of Biomedical Materials 4:153-161.

Van der Rijt, J.A.J., K.O. van der Werf, M.L., Bennink, P.J. Dijkstra, and J. Feijen. 2006. Micromechanical testing of individual collagen fibrils. Macromolecular Bioscience 6(9): 697-702.

Vashishth, D. 2007. The role of collagen matrix in skeletal fragility.Current Osteoporosis Reports 5:62-66.

Vashishth, D., Wu, P., Gibson, G. 2004. Age-related loss in bone toughness is explained by non-enzymatic glycation of collagen. Transactions of the Orthopaedic Research Society 29:497.

Viguet-Carrin, S., Garnero, P., Delmas, D.P. 2006. The role of collagen in bone strength. Osteoporosis International 17:319-336.

Wagner, H.D.,Weiner, S. 1992. On the relationship between the microstructure of bone and its mechanical stiffness. J. Biomech. 25:1311-1320.

Walsh, W.R., Guzelsu, N. 1994. Compressive properties of cortical bone: mineral-organic interfacial bonding.” Biomater 15: 137-145.

Wang, X., Shen, X., Li, X., Agarwal, C.M. 2002. Age-related changes in the collagen network and toughness of bone. Bone 31: 1-7.

Wang, X.D., Puram, S. 2004. The toughness of cortical bone and its relationship with age. Ann. Biomed Eng. 32:123-135.

Weiner, S., Wagner, H.D. 1998.The material bone. Structure-mechanical function relations. Annu. Rev. Material Sci 28: 271-298.

Wu, P., Koharski, C.,Nonnenmann, H., Vashishth, D. 2003. Loading on non-enzymatically glycated and damaged bone results in an instantaneous fracture. Transactions of the Orthopaedic Research Society 28:404.

Yuan, F., Stock, SR., Haeffner, DR., Almer, JD., Dunand, DC., Brinson, L.C. 2011. A new model to simulate the elastic properties of mineralized collagen fibril.” Biomech Model Mechanobiol 10:147-160. 


\section{TABLE CAPTIONS}

Table 1 - Uniaxial failure properties of hydroxyapatite and collagen.

Table 2 - HA mineral volume fractions reported in the literature. 


\section{FIGURE CAPTIONS}

Figure 1 - Microfibril structure: (a) Illustration of assembling TC molecules and the mineral crystals (adapted from Rho et al., 1998), (b) Double period model of cylindrical microfibril composed of (i) five TC molecules shifted by the interval D forming a cylindrical shape, (ii) mineral phase filling the gap space and the extra-collagenous space, and (iii) Cross-links joining two TC molecule ends (Hambli and Barkaoui, 2012).

Figure 2 - Toughness vs. stiffness diagram for bone and biological materials mainly composed of TC and HA (adapted from Yuan et al., 2011).

Figure 3 - Collagen cross-links: (a) Location of enzymatic cross-links (Bailey et al., 1998), (b) finite element modelling of cross-links, (c) Rheological model of the non-linear spring element representing a cross-link behaviour with three regimes: (i) elastic behaviour, (ii) delayed spring response and (iii) friction due to the intermolecular slippage (adapted from Uzel and Buehler, 2011).

Figure 4 - Elastic energy and damping capacity.

Figure 5 - Microfibril tensile stress-strain behaviour at small strain regime for different models and TC states (dry and wet): predicted 3D FEM and experimental results (SAX) from (Sasaki and Odajima, 1996), by atomic force microscopy (AFM) from (Van der Rijt et al. 2006; Aladin et al., 2010) and molecular dynamics (MD) computation from (Li et al., 2003).

Figure 6 - Fracture stress of a collagen microfibril as a function of the cross-link number under different HA mineral Young's modulus (Em).

Figure 7 - Damping coefficient of a collagen microfibril as a function of the cross-link number $(\mathrm{N})$ under different mineral fraction volumes (Vm).

Figure 8 - FEM Von Mises stress $\left(\mathrm{N} / \mathrm{nm}^{2}\right)$ contours under different mineral cross-link numbers $(\mathrm{N})$ and $E_{m}=114 \mathrm{GPa}, E_{c}=2.7 \mathrm{GPa}$ and $V_{m}=48 \%$. 
Figure 9 - Fracture stress of a collagen microfibril as a function of the cross-link number and varying HA mineral volume fraction (Vm).

Figure 10 - Damping coefficient of a collagen microfibril as a function of the HA mineral volume fraction with different cross-link numbers $(\mathrm{N})$.

Figure 11 - Mineralized collagen microfibril crack initiation for different number of crosslinks (N). 
TABLES

Table 1

\begin{tabular}{ccc}
\hline $\begin{array}{c}\text { Uniaxial tensile } \\
\text { strength (MPa) }\end{array}$ & $\begin{array}{r}\text { Uniaxial shear } \\
\text { strength (MPa) }\end{array}$ \\
\hline HA Crystal & $\sigma_{H A}^{\text {ult,t }}=52.2$ & $\sigma_{H A}^{\text {ult,s }}=80.3$ \\
TC molecules & $\sigma_{c o}^{\text {ult, }=144}$ & NA \\
\hline
\end{tabular}

Table 2

Reference

Currey, 1969

Lees, 1987

Sasaki et al., 1991

Wagner and Weiner, 1992

Jager and Fratzl, 2000

Kotha and Guzelso, 2000

Ji and Gao, 2000

Nikolov and Raabe, 2008
HA mineral volume fraction (\%)

50

45

50

35

43

50

45

52 


\section{FIGURES}

Figure 1

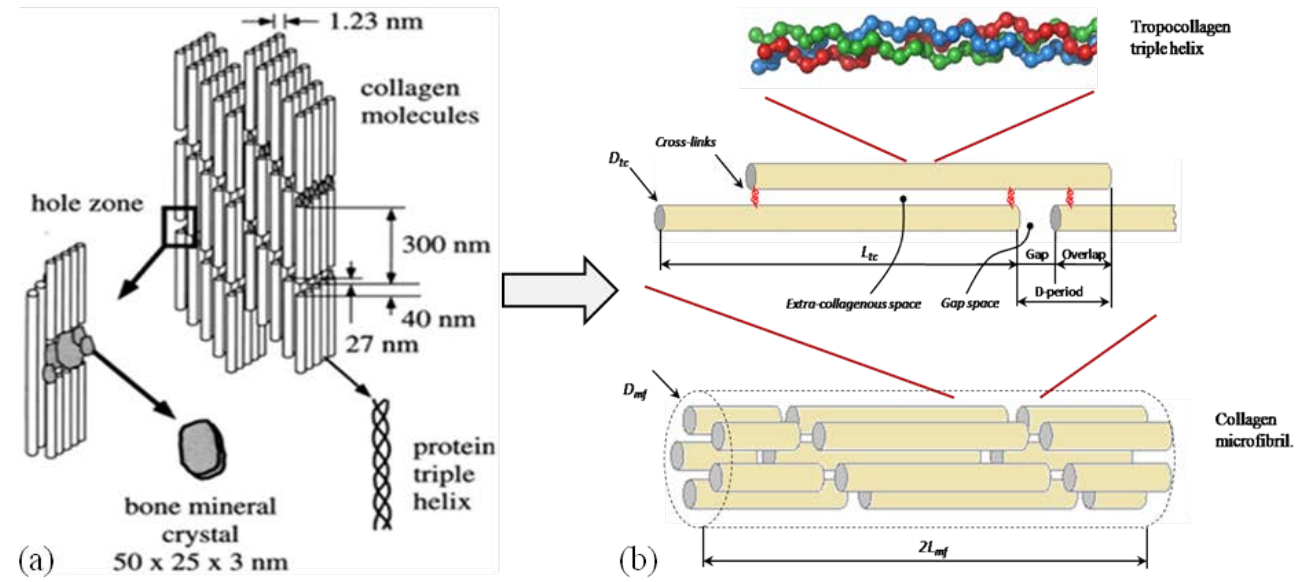

Figure 2

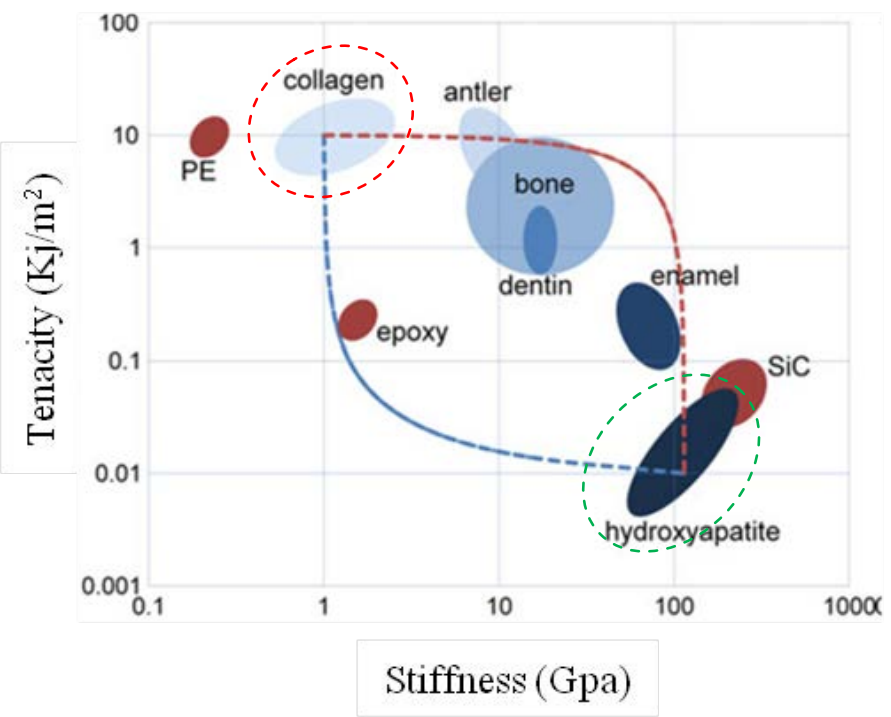


Figure 3

(a)

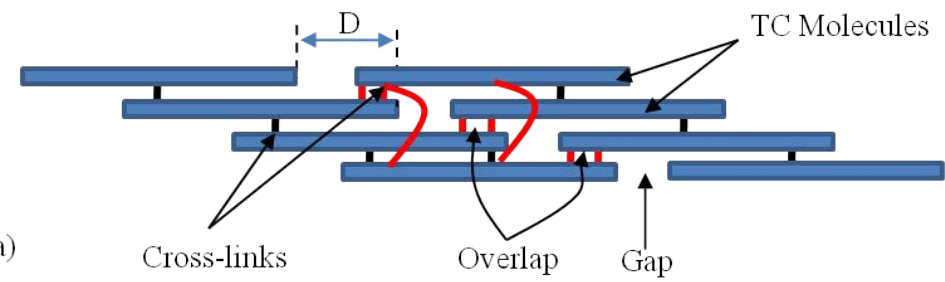

(b)

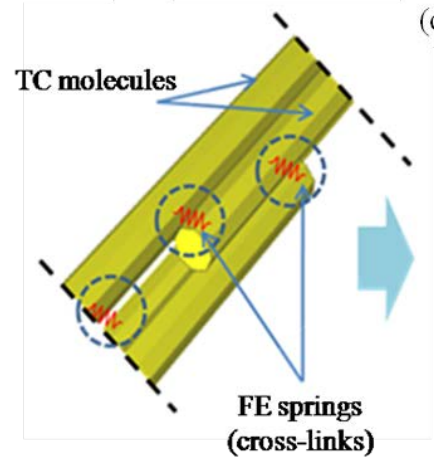

(c)

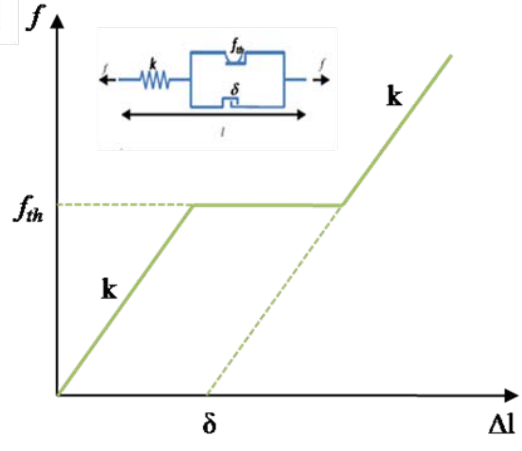

\section{Figure 4}

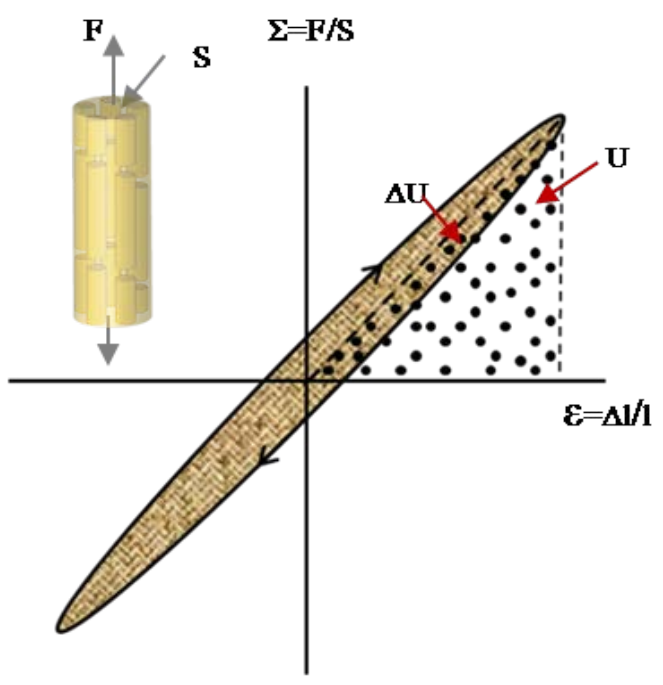


Figure 5

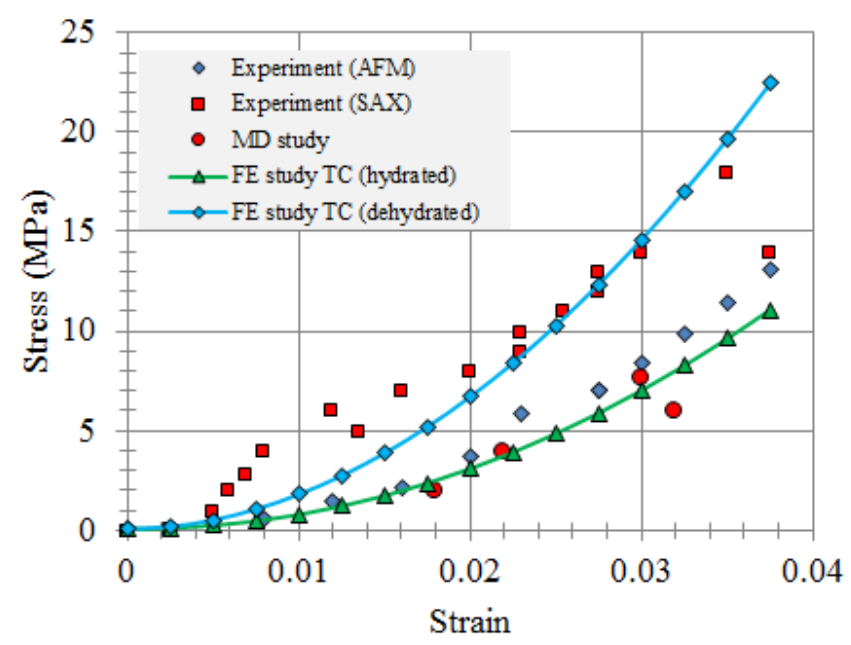

Figure 6

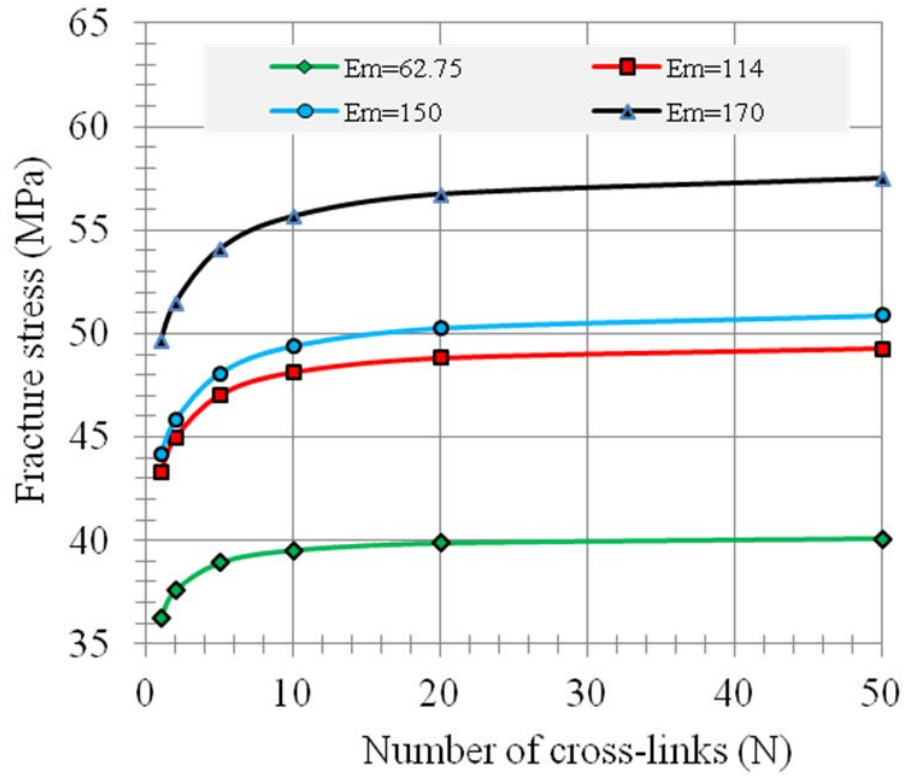




$$
\equiv
$$


Figure 9

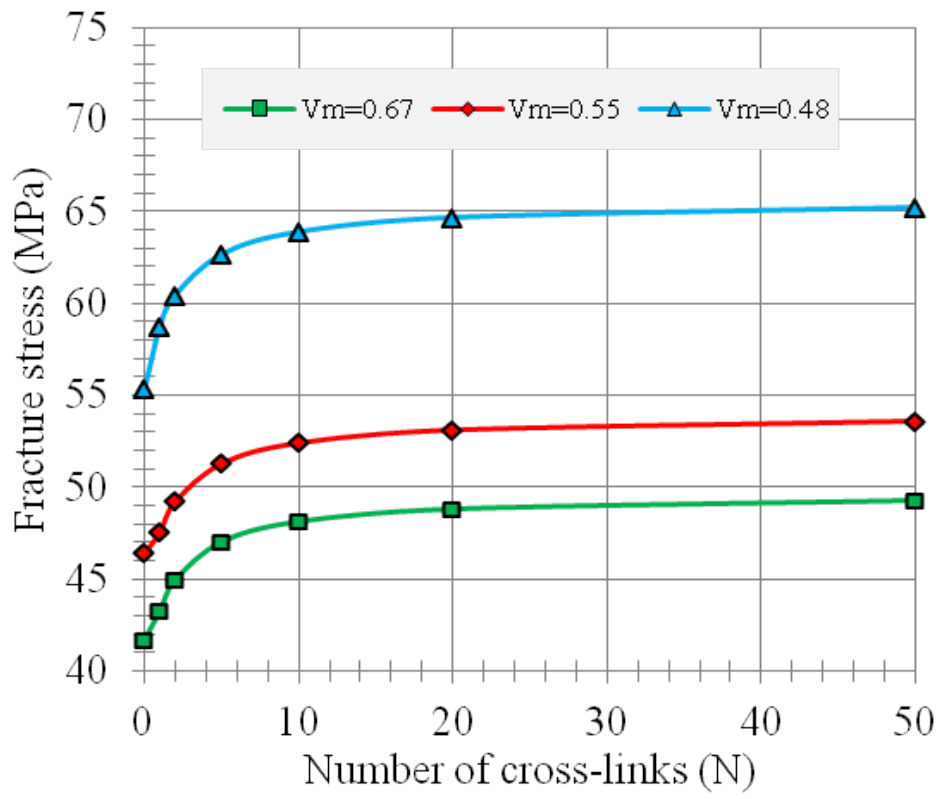

Figure 10

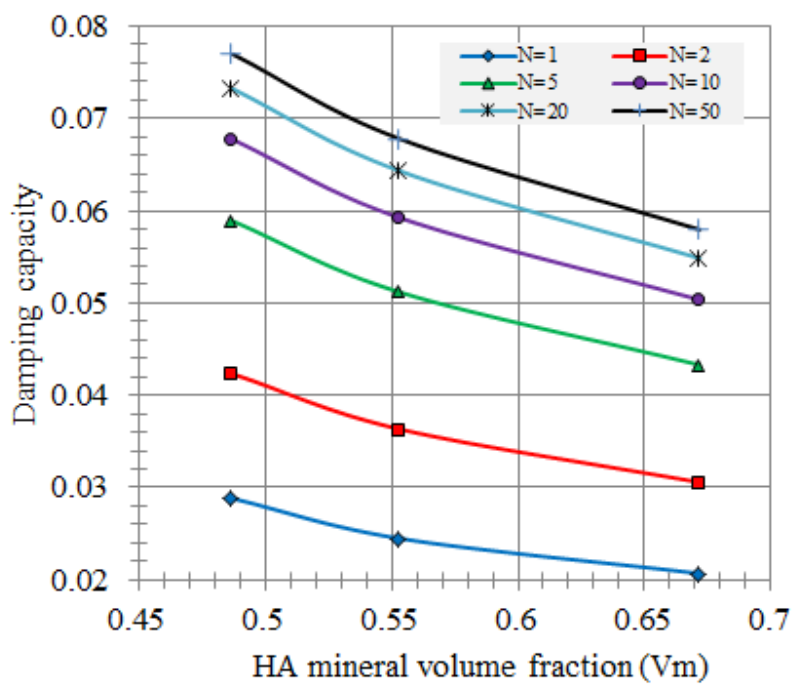


Figure 11

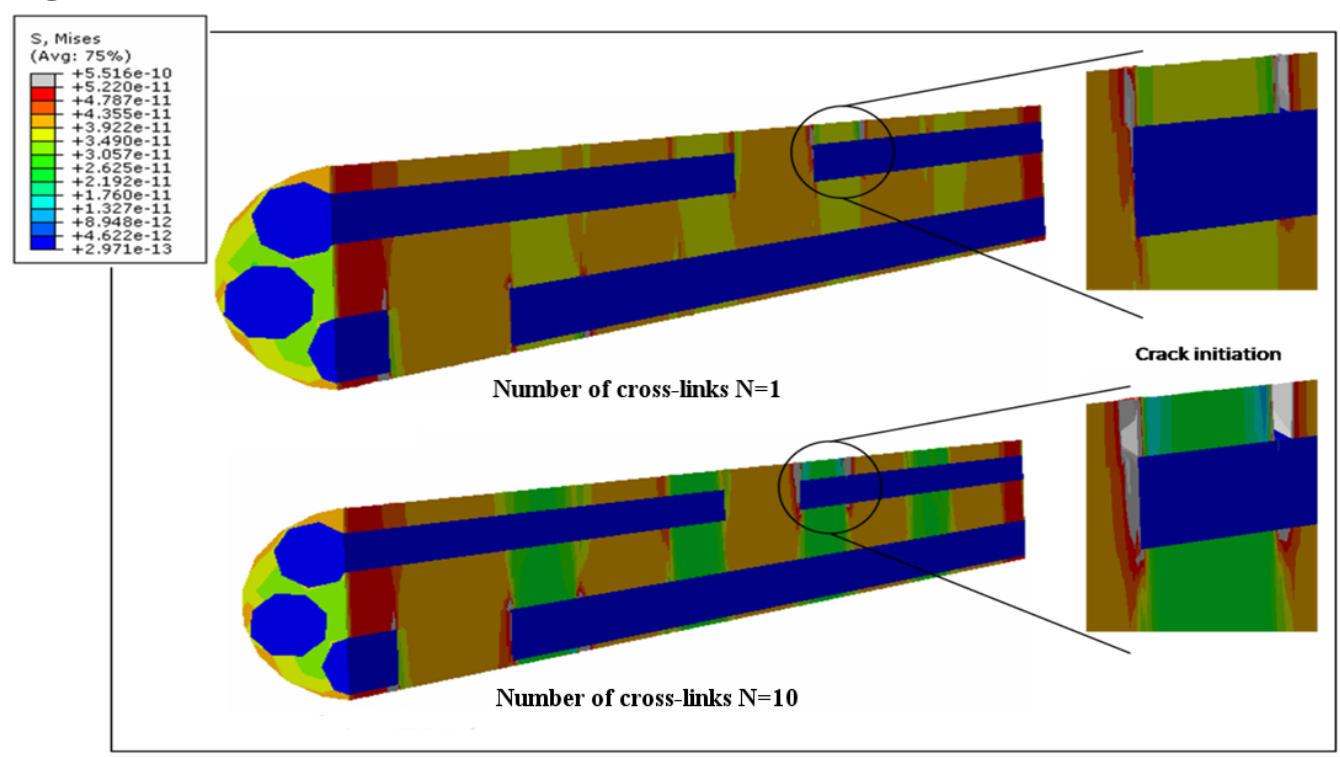

